\title{
Semiflexible polymer conformation, distribution and migration in microcapillary flows
}

\author{
Raghunath Chelakkot ${ }^{\dagger}$, Roland G. Winkler ${ }^{\ddagger}$, Gerhard \\ Gompper $^{\dagger, \ddagger}$ \\ ${ }^{\dagger}$ Institut für Festkörperforschung and ${ }^{\ddagger}$ Institute for Advanced Simulation, \\ Forschungszentrum Jülich, 52425 Jülich, Germany
}

\begin{abstract}
The flow behavior of a semiflexible polymer in microchannels is studied using Multiparticle Collision Dynamics (MPC), a particle-based hydrodynamic simulation technique. Conformations, distributions, and radial cross-streamline migration are investigated for various bending rigidities, with persistence lengths $L_{p}$ in the range $0.5 \leq L_{p} / L_{r} \leq 30$. The flow behavior is governed by the competition between a hydrodynamic lift force and steric wallrepulsion, which lead to migration away from the wall, and a locally varying flow-induced orientation, which drives polymer away from the channel center and towards the wall. The different dependencies of these effects on the polymer bending rigidity and the flow velocity results in a complex dynamical behavior. However, a generic effect is the appearance of a maximum in the monomer and the center-of-mass distributions, which occurs in the channel center for small flow velocities, but moves off-center at higher velocities.
\end{abstract}




\section{Introduction}

\subsection{Polymers, Vesicles and Cells in Micro- and Nanochannels}

Complex fluids - such as polymer solutions, colloidal dispersions, and suspensions of vesicles or cells - exhibit an intriguing flow behavior, particularly in confined geometries. The interplay of internal degrees of freedom, e.g, conformational changes of polymers or shape changes of vesicles and cells, combined with fluid mediated interactions, inhomogeneous flow profiles, and wall interactions lead to novel and often unexpected effects [1 18. Insight into theses aspects provides not only an understanding of phenomena for conventional applications such as lubrication, adhesion, polymer processing, and blood flow, but also of emergent microfluidic devices [19, 20 and the lab-on-a-chip technologies 21] with their micro- or nano-size length scale. In this context, the investigation and analysis of single molecules or cells is of particular interest, since on the one hand such knowledge is essential to develop and optimize micron- and nano-scale devices, while on the other hand the study of individual particles often provides a more detailed microscopic knowledge than can be extracted from an ensemble.

The size of red blood cells (RBCs), about $8 \mu \mathrm{m}$ in diameter, is comparable to the size of microvessels and microcapillaries in a mammalian body. Thus, the deformability and dynamics of an individual cell determines the flow behavior of blood in the capillary network. Due to the physiological importance of this process and the relatively large size of the objects, detailed investigations of this system started already about 50 years ago $[22] 25$. Modern microfluidic techniques [20,26]27] and new mesoscale simulation approaches 2831 provide new insight into the delicate interplay between flow forces, cell deformation, and hydrodynamic interactions $10,17,32,33$. In particular, in microchannels slightly larger than the RBC diameter, it has been found that single red blood cells transform from their equilibrium shape of biconcave disks into a parachute shape at a critical flow velocity, which depends linearly on the bending rigidity and the shear modulus of the cell membrane [10. At small volume fractions of red blood cells, they show a strong clustering tendency due to hydrodynamic interactions [17]. At higher volume fractions, several distinct phases are found, which range from disordered arrangement of discocytes over single-file motion of aligned parachutes to a staggered, zig-zag arrangement of slipper shapes [17. In channels which are smaller than the RBC diameter, they assume bullet shapes at sufficiently high flow velocities $23,32,33$.

Fluid vesicles are distinct from red blood cells since their membrane is fluid, and therefore has a vanishing shear modulus. The flow behavior of vesicles has been studied intensively in recent years, in particular in shear flows. Much less is known about fluid vesicles in capillary flows. In narrow, homogeneous channels, vesicles assume bullet shapes 34. In structured, saw-tooth shaped channels, which are somewhat larger than the vesicle size, a complex conformational and dynamical behavior has been found [18. For nearly spherical vesicles, a transition from symmetric shape oscillations to orientational oscillations has been predicted with decreasing flow velocity. For shapes, which deviate more strongly from a sphere, experiments and simulations show new shapes with two symmetric or a single asymmetric tail [18.

In narrower channels, with a diameter on the order of $100 \mathrm{~nm}$, it is possible to investigate the static and dynamic behavior of single DNA molecules. This system is interesting for several reasons. First, microfluidic devices allow for the manipulation, 
sizing, and sorting of DNA fragments [35, 36], and thereby a direct visualization of genomic information [7,37. Second, DNA has turned out to be an ideal model to study the properties of single polymers, because, when fluorescently labelled, its conformations, dynamics, and flow properties can be observed directly under a microscope [1,38, 42] or by correlation spectroscopy [43. Thus, DNA is also very well suited to study the behavior of semiflexible polymers in micro- and nanochannels.

With a persistence length of $L_{p} \simeq 17 \mu m$, filamentous actin is a biopolymer for which semiflexible behavior becomes important already in wider channels than for DNA (with its persistence length $L_{p} \simeq 50 \mathrm{~nm}$ ). Thus, experiments with actin are essential to elucidate the behavior of semiflexible polymers in microchannels without [44 46] and with flow [47. In equilibrium, the parallel extension of a semiflexible polymer shows scaling behavior with power-law dependencies on channel diameter and persistence length [40,48,51].

Structured channels are particular interesting, because they allow to study timedependent flows [18, threshold forces or flow rates for penetration into narrow pores 16, 52 54, and flow injection into a pore 16. For fluid vesicles, it has been shown that the threshold strength of a driving field (e.g., an electric field) for narrow pores increases nearly linearly with membrane bending rigidity and vesicle area, and decreases rapidly with the pore radius [52]. For polymers, it has been predicted by scaling arguments [53] and confirmed by simulations [16] that the threshold velocity flux for entry into a narrow pore is independent of both the polymer length and the pore radius.

\subsection{Polymer Migration in Micro- and Nanochannels}

An interesting aspects of polymer transport in the presence of a zero-slip wall is cross-streamline migration. This effect has been observation in planar shear flow in the presence of walls as well as Poiseuille flows [4, 6, 9, 11, 12, 14, 15, 55, and in experiments [5,7,13, as a formation of depletion layer near the wall. The thickness of the depletion layer is found to increase with flow, which indicates that migration away from the wall increases with the flow rate. This migration phenomenon is explained as a results of polymer-wall hydrodynamic interactions. Analytical as well as simulation studies, which properly account for such hydrodynamic interactions, qualitatively reproduced the experimentally observed wall-induced migration.

In microchannels, confinement effects and a spatially varying shear rate due to the parabolic flow velocity profile imply additional features compared to simple shear flows. The steric interaction with the channel wall restricts the conformations of a flexible polymer and induce alignment to a semiflexible polymer. Moreover, the spatially varying local shear rate changes the conformations and alignment of a polymer as a function of its lateral position. In addition to the wall hydrodynamic interactions, these two effects also influence the qualitative behavior of cross-streamline migration. Simulations of flexible polymers have shown that under strong confinement there is a net migration away from the channel center, contrary to the predictions of wall induced inward migration 11. The outward migration is also found in relatively weak flow [15. In both cases, the outward migration has been attributed to the suppression of steric interactions with the wall due to an enhanced alignment by the flow. At large flow strengths, wall hydrodynamic interactions dominate, resulting a net inward migration of the polymer center-of-mass. Interestingly, the center-of-mass density at the channel center is also found to decrease with flow and at large flow 
strengths a density maximum is found at a distance away from the center. The reason behind the off-center peak of the center-of-mass distribution for flexible polymers is the flow-induced conformational change, which leads to stretched polymers close to the wall and coiled chains in the central part [4,6, 9, 11, 12, 14, 15. This give rise to an enhanced outward diffusion and ultimately leads to a concentration dip at the center.

Although the cross-streamline migration behavior of flexible polymers has been extensively studied, there are only a few studies, which focus on the migration behavior of semiflexible and rodlike polymers [56,57. Understanding the migration properties of semiflexible and stiff polymers is important for the study of many biopolymers, such as actin filaments, microtubules, and intermediate filaments. Since polymer conformations are determined by its rigidity, the migration behavior of semiflexible and rodlike polymers can differ significantly from that of flexible polymers.

In this paper, we use computer simulations to study and discuss the migration properties of semiflexible polymers with persistence length ranging from half up to many times of its contour length. We employ a hybrid simulation scheme including Molecular Dynamics (MD) for the polymers and Multiparticle Collision Dynamics (MPC), a particle-based hydrodynamics simulation technique, for the solvent, so that wall hydrodynamic interactions and confinement effects are taken into account explicitly. The details of the method are given in Sec. 2. Our results, presented in Secs. 3 and 4 , show that polymer migration is indeed qualitatively influenced by bending rigidity. Furthermore, in Sec. 5. we investigate the importance of hydrodynamic interactions by comparing the results of simulations with MPC and Brownian solvents. Finally, the dynamics of polymer migration across the channel is studied in Sec. 6

\section{Model and Simulation Method}

\subsection{Semiflexible Polymer}

The linear polymer is comprised of $N_{m}$ point-like monomers of mass $M$ each, which are connected by the harmonic potential

$$
U_{s}=\frac{\kappa_{s}}{2} \sum_{i=1}^{N_{m}-1}\left(\left|\boldsymbol{r}_{i+1}-\boldsymbol{r}_{i}\right|-b\right)^{2}
$$

where $r_{i}$ denotes the position of particle $i, b$ the bond length, and $\kappa_{s}$ the force

constant. Excluded-volume interactions are taken into account by the Lennard-Jones potential [58]

$$
U_{L J}=\left\{\begin{array}{ll}
4 \epsilon\left[\left(\frac{\sigma}{r}\right)^{12}-\left(\frac{\sigma}{r}\right)^{6}\right]+\epsilon, & r<2^{1 / 6} \sigma \\
0, & \text { else }
\end{array} .\right.
$$

To account for polymer stiffness, the bending potential is applied [59]

$$
U_{b}=\frac{\kappa_{b}}{2} \sum_{i=2}^{N_{m}-1}\left(\boldsymbol{R}_{i+1}-\boldsymbol{R}_{i}\right)^{2},
$$

where $\boldsymbol{R}_{i}=\boldsymbol{r}_{i}-\boldsymbol{r}_{i-1}$ is the bond vector and $\kappa_{b}$ is the bending rigidity. In the semiflexible limit, the bending rigidity is related to the persistence length by $L_{p}=\kappa_{b} / k_{B} T$.

The dynamics of the polymer is described by Newtons' equations of motion, which are integrated using the velocity-Verlet algorithm. 


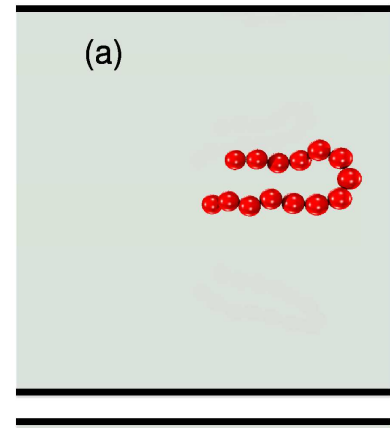

(d)

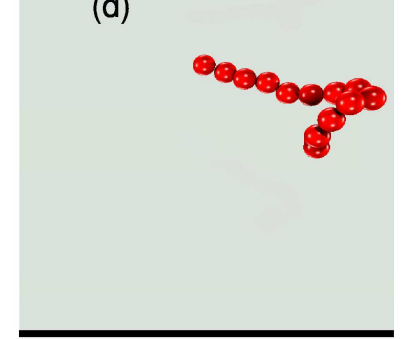

(b)

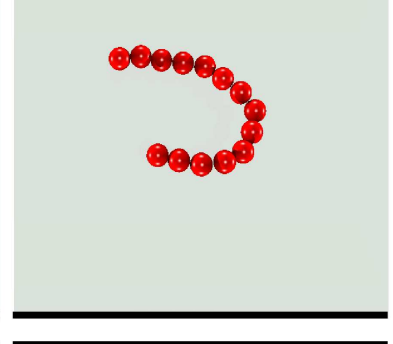

(e)

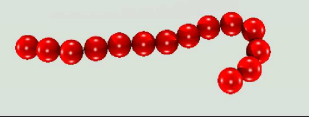

(c)

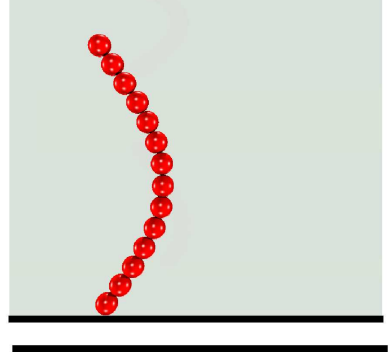

(f)

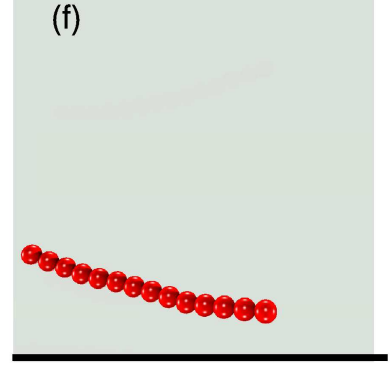

Figure 1. Snapshots of polymer conformations close to the channel center (top) and the wall (bottom) for the Peclet number $P e=360$ and the persistence lengths $L_{p} / L_{r} \approx 0.5$ (a), (d), 2.1 (b), (e), and 30.8 (c), (f). Movies are available as Supporting Material 60.

\subsection{Hydrodynamic Solvent}

In the MPC algorithm, the fluid is described by a set of $N$ point-like particles of mass $m$ each, which move in continuous space with velocities determined by a stochastic process. Their dynamics evolves in two steps. In the streaming step, the solvent particles move ballistically for a time $h$, which we denote as collision time. In the collision step, particles are sorted into the cells of a cubic lattice of lattice constant $a$ and their relative velocities, with respect to the center-of-mass velocity of each cell, are rotated around a random axis by an angle $\alpha$. The orientation of the axis is chosen independently for every cell and collision step. For every cell, mass, momentum, and energy are conserved in this process. The algorithm is described in detail in refs. 61 66. The fluid is confined in a cylindrical channel with periodic boundary conditions along the channel axis. No-slip boundary conditions are imposed on the channel walls by the bounce-back rule and virtual wall particles, as described in ref. 67. The flow is induced by a gravitational force $(\mathrm{mg})$ acting on every fluid particle.

The interaction of a polymer with the solvent is realized by inclusion of its monomers in the MPC collision step 68. Between two MPC steps, several MD steps are performed to update the positions and velocities of the monomers. Extensive studies of polymer dynamics confirm the validity of this procedure [58,66, 68, 69,

To maintain a constant temperature, the velocity scaling algorithm is applied 
as described in Ref. [70]. Here, an kinetic energy $E_{k}$ is chosen from the gamma distribution independently for every cell, and the individual particle velocities of that cell are multiplied by the factor $\left(2 E_{k} /\left(m \sum \boldsymbol{v}_{i}\right)\right)^{1 / 2}$, where the sum runs over all particles in the considered cell. This assures that the velocity distribution of the particles is Maxwellian. Similarly, a Maxwellian velocity distribution is obtained by the Monte Carlo procedure described in Ref. [71].

Simulations of the pure solvent system yield velocity profiles which agree with the solution of Stokes' equation for the considered geometry.

\subsection{Brownian Solvent}

An advantage of the MPC approach is that hydrodynamic interactions can easily be switched off, without altering the monomer diffusion significantly 72 , 73. In this case, denoted as Brownian MPC, each monomer independently performs a stochastic collision with a phantom particle with a momentum taken from the MaxwellBoltzmann distribution with variance $m\left\langle N_{c}\right\rangle k_{B} T$, where $\left\langle N_{c}\right\rangle$ is the average number of solvent particles per collision cell [73].

\subsection{Parameters}

For the solvent, we employ the parameters $\alpha=130^{\circ}, h=0.1 \tau$, with $\tau=\sqrt{m a^{2} / k_{B} T}$ ( $k_{B}$ is Boltzmann's constant and $T$ is temperature), $\left\langle N_{c}\right\rangle=10, M=m\left\langle N_{c}\right\rangle, b=\sigma=$ $a$, the fluid mass density $\varrho=\left\langle N_{c}\right\rangle m / a^{3}$, and $k_{B} T / \epsilon=1$. The time step in the MD simulation is set to $h_{M D}=5 \times 10^{-3} \tau$. A polymer with $N=14$ monomers is placed in a cylindrical channel of radius $R=8 a$. With the length $L_{r}=(N-1) a=13 a$, the polymer does not interact with the wall when its center of mass is near the channel center. In order to maintain a constant contour length of the polymer, we set $\kappa_{s}=3 \times 10^{3} /\left(k_{B} T / b^{2}\right)$. The persistence length $L_{p}$ is varied, by selecting the values $\kappa_{b} /\left(k_{B} T / b^{2}\right)=7,28,50,100,200,400$, which correspond to the persistence lengths $L_{p} / L_{r} \approx 0.5,2.1,3.8,7.7,15.4,30.8$. The channel length is $28 a$.

The strength of the applied pressure field is characterized by the Peclet number $P e=\dot{\gamma} \tau_{R}$, where $\dot{\gamma}=g \varrho R /(2 \eta)$ is the shear rate at the cylinder wall. The Reynolds number $R e=\varrho R v_{m} / \eta=\varrho R^{2} \dot{\gamma} /(2 \eta)$, where $v_{m}$ is the maximum fluid velocity, depends linearly on the shear rate. For the above MPC parameters, the viscosity [65] is such that $R e<1$ for all considered $\dot{\gamma}$. Equilibrium simulations for a system with periodic boundary conditions yield the end-to-end vector relaxation time $\tau_{R} \approx 3200 \tau$. This value agrees within approximately $20 \%$ with the relaxation time obtained theoretically for a semiflexible polymer with the same ratio $L_{r} / L_{p}$ 74. The relaxation time of the Brownian MPC simulation is $\tau_{R} \approx 8200 \tau$.

The averages and probability distributions presented in the following sections are calculated in the stationary state for various independent initial conditions.

\section{Radial Distribution Functions}

The imposed flow, with a parabolic flow profile, determines the polymer conformations, with a few examples shown in fig. 1, and the polymer distribution functions, such as the monomer distribution $P_{m o}$ and the center-of-mass distribution $P_{c m}$. Their dependence on flow strength will be characterized in the following sections. 


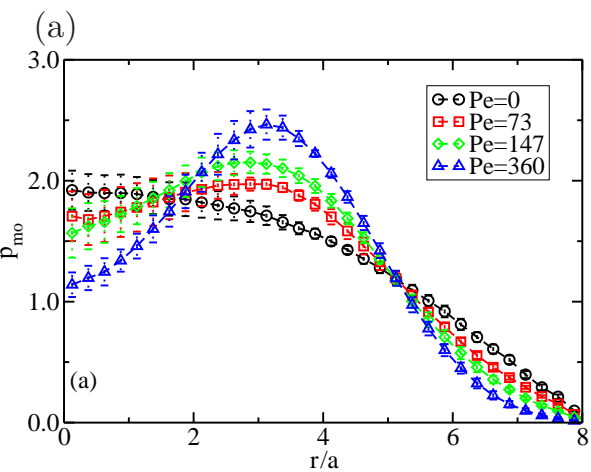

(c)

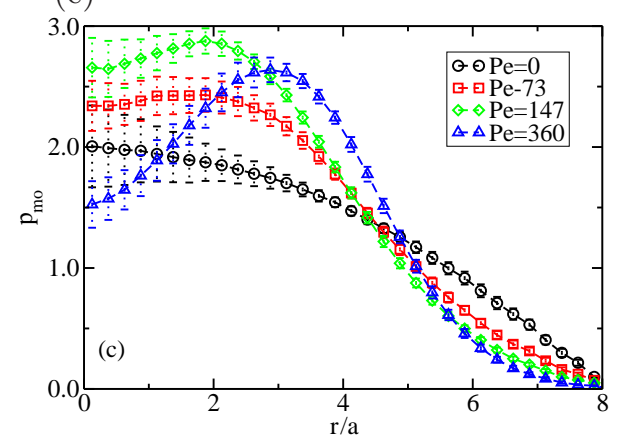

(b)

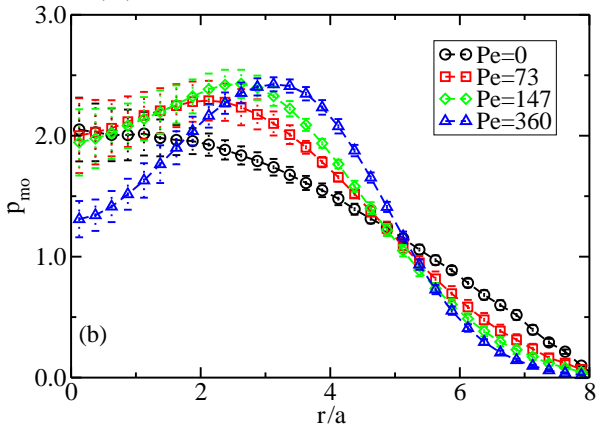

(d)

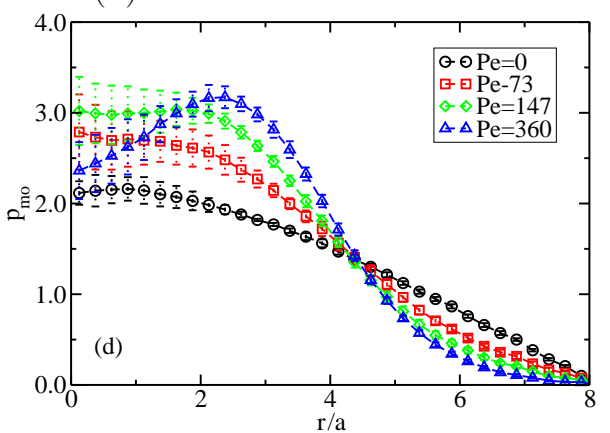

Figure 2. Radial monomer distributions for the Peclet numbers $P e=0$ (०), 73 $(\square), 147(\diamond)$, and $360(\triangle)$ of polymers with $L_{p} / L_{r} \approx 3.8(\mathrm{a}), 7.7$ (b), 15.4 (c), and $30.8(\mathrm{~d})$.

\subsection{Radial Monomer Distribution}

Radial monomer distributions are presented in fig. 2 for various flow rates and stiffnesses. They are normalized such that

$$
\int_{0}^{R / a} r P_{m o}(r / a) d r / a^{2}=1
$$

A feature in common with all polymer systems with hydrodynamic interactions is the decrease of the monomer concentration adjacent to the wall with increasing flow rate. Even without flow, there is a depletion zone for all stiffnesses, which extends approximately one radius of gyration into the channel. However, the distributions exhibit distinct differences to those obtained previously for flexible and rodlike polymers, respectively. For persistence lengths $L_{p} / L_{r} \lesssim 8$, the density at the center of the channel decreases with increasing Peclet number as shown in figs. 2ha,b. In contrast, for stiffer polymers with $L_{p} / L_{r}>8$ the density at the channel center first increases with increasing $P e$ and then decreases at large Peclet numbers. Simultaneously, the wall induced migration towards the channel center causes an increase in concentration at a finite distance from the center for all stiffnesses. Two effects contribute to the formation of the maximum. On the one hand, there is cross-streamline migration due to hydrodynamic interactions, and, on the other hand, the flow field causes an alignment of a molecule (see section 4), which increases the local density at the radial 


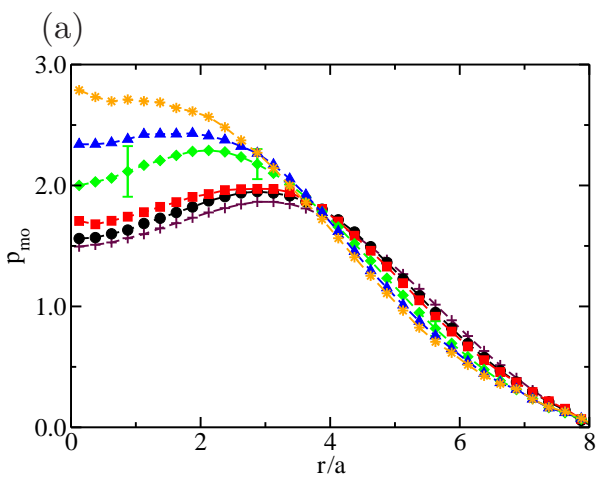

(b)

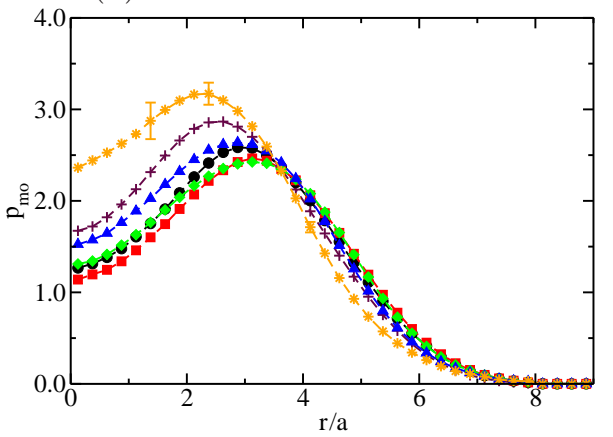

Figure 3. Radial monomer distributions for the Peclet numbers $P e=73$ (a) and 360 (b) and the persistence lengths $L_{p} / L_{r} \approx 0.5(+), 2.1(\bullet), 3.8(\square), 7.7(\diamond)$, $15.4(\triangle)$, and $30.8(\star)$.

positions of strong flow-induced alignment.

The distribution functions are rather similar for the various stiffnesses at $P e=0$ within the accuracy of the simulation. Interestingly, stiffer polymers exhibit a more pronounced migration away from the wall for $P e \lesssim 100$ as is shown in fig. 3 $\mathrm{k}$. Simultaneously, $P_{m o}$ decreases in the channel center for small stiffnesses, leading to an off-center maximum, and increases for larger ones, where the maximum is at the center for some of the larger stiffnesses. With increasing flow rate, off-center density maxima are obtained for all stiffnesses. For the flow rate $P e=360$, we observe a decrease in the depletion zone adjacent to the wall with increasing stiffness for $L_{p} / L_{r}<8$ and a reversion of the trend at larger stiffnesses as depicted in fig. $3 \mathrm{~b}$.

The width of the distribution,

$$
\left\langle r^{2}\right\rangle=\int_{0}^{R / a} r^{3} P_{m o}(r / a) d r / a^{2},
$$

emphasizes the differences in migration behavior. As displayed in fig. [6], stiffer polymers exhibit a smaller width at moderate Peclet numbers, indicating an enhanced net inward migration with increasing bending rigidity, but $\left\langle r^{2}\right\rangle$ seems to saturate at large flow rates. In contrast, the widths of more flexible polymers show only minor changes at small $P e$ and decrease more rapidly at larger Peclet numbers. Their widths can be smaller than those of stiffer polymers.

\subsection{Radial Center-of-Mass Distribution}

Radial polymer center-of-mass distributions are displayed in fig. (4) Qualitatively, $P_{c m}$ exhibits similar features as the monomer distribution $P_{m o}$, reflecting the same physical mechanisms. For $L_{p} / L_{r} \lesssim 8$, the concentration at the channel center decreases with increasing $P e$. The density profiles for $50 \lesssim P e \lesssim 150$ are very similar and indicate a weak dependence of the center-of-mass properties on the flow rate only. For high $P e$ values, the densities at the channel center decrease significantly for all stiffnesses, and a clear maximum appears at $r_{c m}>0$. The density decrease with flow near the wall is mainly due to wall induced cross-streamline migration of polymers and partly due to steric interactions of the very stiff polymers with the wall, while the density 


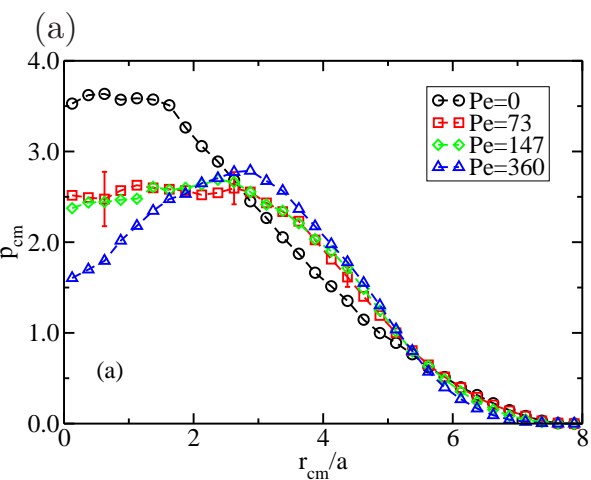

(b)

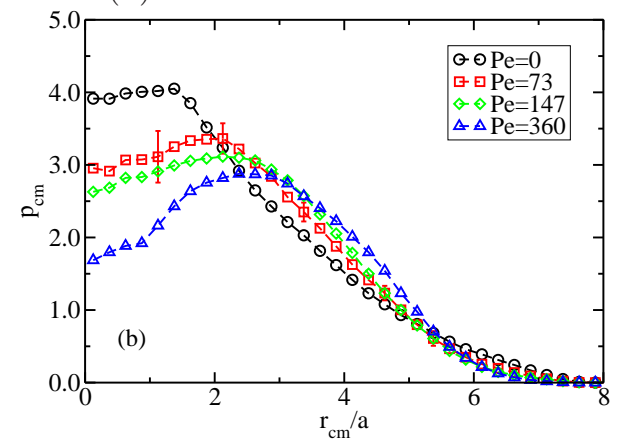

(c)

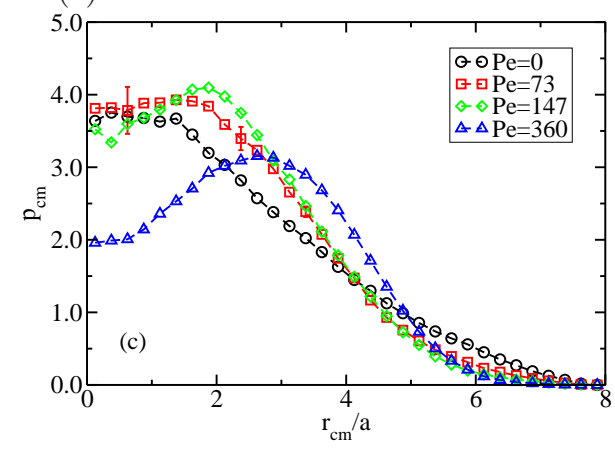

(d)

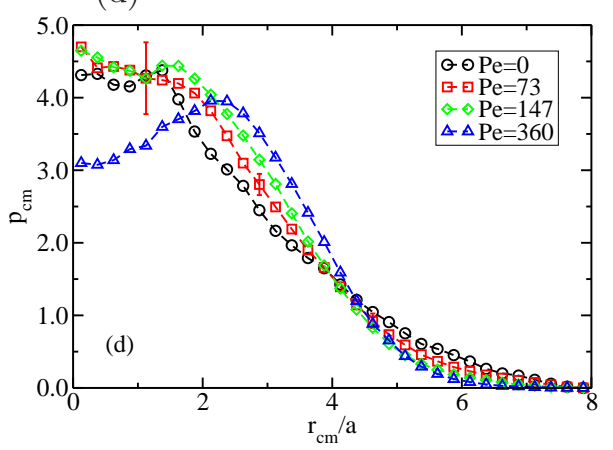

Figure 4. Radial center-of-mass distributions for the Peclet numbers $P e=0$ (०), $73(\square), 147(\diamond)$, and $360(\triangle)$ of polymers with $L_{p} / L_{r} \approx 3.8$ (a), 7.7 (b), 15.4 (c), and $30.8(\mathrm{~d})$.

(a)

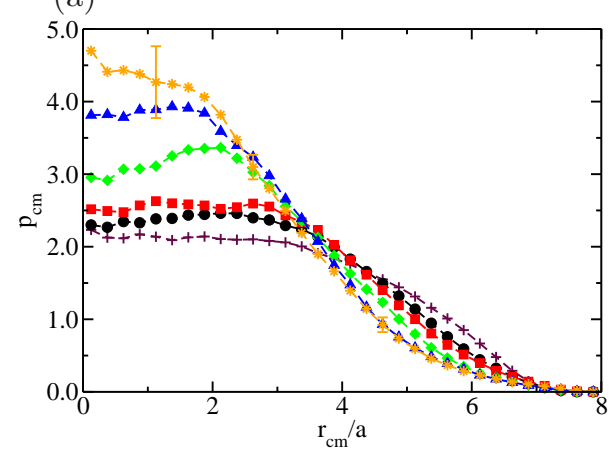

(b)

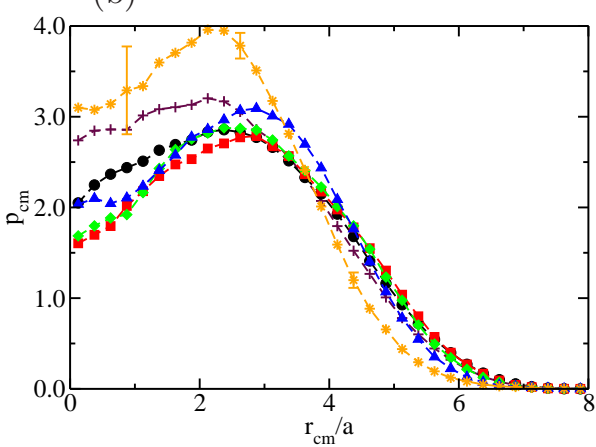

Figure 5. Radial center-of-mass distributions for the Peclet numbers $P e=73$ (a) and $360(\mathrm{~b})$ and persistence lengths $L_{p} / L_{r} \approx 0.5(+), 2.1(\bullet), 3.8(\square), 7.7(\diamond)$, $15.4(\triangle)$, and $30.8(\star)$. 

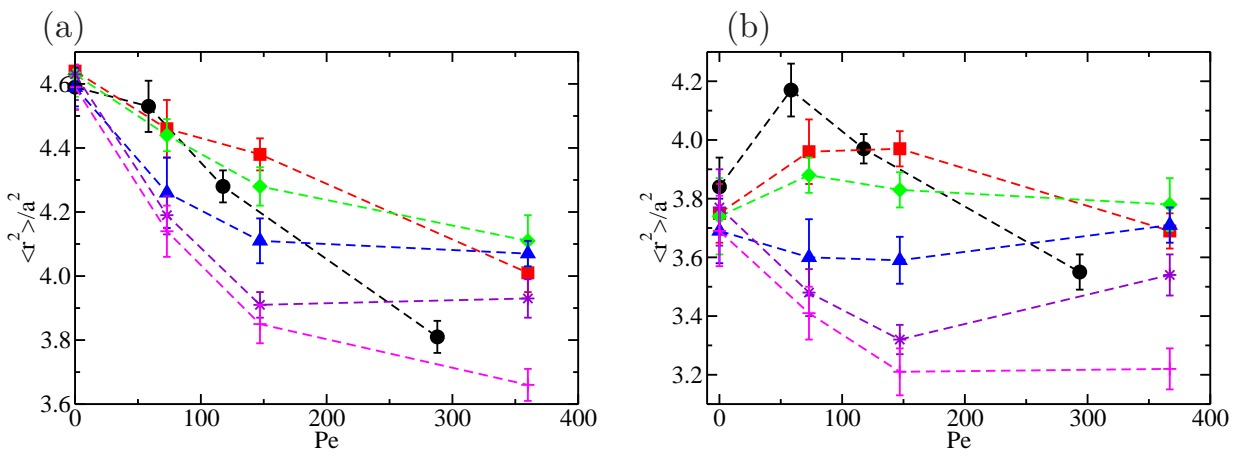

Figure 6. Widths $\left\langle r^{2}\right\rangle$ of the radial monomer distributions (a) and center-ofmass distributions $(\mathrm{b})$ for the persistence lengths $L_{p} / L_{r} \approx 0.5(\bullet), 2.1(\square), 3.8$ $(\diamond), 7.7(\triangle), 15.4(\star)$, and $30.8(+)$.

decrease at the channel center indicates a migration away from the center. The steric contribution to migration is evident from the Brownian MPC simulations presented in sec. 5. The two competing migration mechanisms result in the formation of a maximum at a distance away from the center at large $P e$. The maximum is less pronounced for the center-of-mass distribution than for the monomer distribution.

Figure 5 shows center-of-mass distributions of polymers with different bending rigidities for $P e \approx 73$ and $P e \approx 360$. As for even smaller Peclet numbers, the distributions exhibit a maximum essentially in the channel center for $P e \approx 73$. An offcenter maximum only appears for larger flow rates, which is accompanied by a density decrease in the channel center. Moreover, a pronounced depletion layer is visible at the wall for large stiffnesses.

Flexible polymers exhibit a pronounced density increase adjacent to the wall with increasing flow rate and before migration sets in, whereas very stiff polymers exhibit an increased depletion layer at the wall with increasing flow rate, which we attribute to steric polymer-wall interactions. This is reflected in the flow rate dependence of the width of the center-of-mass distribution displayed in fig. 6b. For $L_{p} / L_{r} \lesssim 4$, the width increases with increasing Peclet numbers for small $P e$. This increase is explained by the suppression of polymer-wall steric interactions due to flow alignment of polymers (cf. section 4). For flexible polymers such an alignment is enhanced by flow-induced polymer stretching along the channel axis. However, at larger $P e$, the wall induced hydrodynamic lift force dominates the dynamics, which results in a decrease of width. This dependence is qualitatively different for polymers with $L_{p} / L_{r} \gtrsim 5$, where wall induced migration dominates even at low $P e$, implying a decrease in width. However, at large Peclet numbers, we observe a saturation or even increase in width by outward migration due to hydrodynamic interactions, which counter balances the radially inward migration, as discussed in Sec. 6.

\section{Alignment and Conformations}

\subsection{Orientational Order Parameter}

In microchannel flows with no-slip boundary conditions the local shear rate changes linearly with radial position. Hence, the flow-induced force experienced by a polymer 
depends on its radial position. Its conformations and alignment, in response to the flow forces, is expected to vary with its bending rigidity and its radial center-of-mass position. Studies of flexible and semiflexible polymers [15.75] reveal large orientational changes by the imposed flow, which is important for cross-streamline migration [55]. To study the influence of flow on the polymer orientation, we consider the orientational order parameter

$$
S\left(r_{c m}\right)=\frac{1}{2}\left\langle 3 \cos ^{2} \theta-1\right\rangle,
$$

where $\theta$ is the angle between the polymer end-to-end vector and the flow direction, as a function of its center-of-mass radial position. $S\left(r_{\mathrm{cm}}\right)$ is plotted for various Peclet numbers and stiffnesses in fig. 7. In the absence of flow and for all $\kappa_{b}$, the polymer orientation is isotropic at the channel center. For distances close to the wall, confinement causes a preferred orientation along the channel axis with $S \approx 1$. With increasing flow rate, the order parameter increases for all stiffness and reaches a plateau at large $P e$ for certain radii. The plateau value and its extension depends on stiffness as shown in fig. 8 .

The order parameter exhibits a non-monotonic dependence on flow strength close to the channel center and depends on polymer stiffness as shown in the inset of fig. 8 An increase in stiffness leads to a decrease of the order parameter for $L_{p} / L_{r}<15$. For larger stiffnesses, $S(0)$ is larger for larger $L_{p} / L_{r}$ at all radial distances. At $P e=360, S$ decreases with increases stiffness and becomes even negative for $L_{p} / L_{r} \simeq 2$, compare the inset of fig. 8 . As the polymer stiffness increases, $S$ increases close to the channel center. The negative values of $S$ at the channel center are attributed to the formation of U-shaped conformations, compare fig. 1. Such conformations are not possible for flexible polymers [15] and polymers with large bending rigidity, hence $S$ assumes higher values at the center as $\kappa_{b}$ increases.

\subsection{Bending Energy}

U-shaped conformations can be quantitatively analyzed by calculating the average bending energy $U_{B}$. Figure 9 displays $U_{b}$ as function of the radial polymer centerof-mass position for various persistence lengths and for various Peclet numbers. For $P e=0$ the bending energy is nearly uniform across the channel cross section and decreases near the wall, due to wall induced alignment. The magnitude of $U_{b}$ is close to the thermal average $U_{b}=\left(L_{p} / a-1\right) k_{B} T$ of the nearly harmonic bending potential for $L_{p} / L_{r}>1$, as expected. An increase in $P e$ results in an increase in its absolute value, which is a result of the conformational changes of the polymer in response to the force exerted by the flow, compare fig. 1. At a given $P e$ and radial position, the flow exerts the same force on a polymer, but its conformations depend on its bending rigidity. Polymers with large bending rigidity hardly undergo conformational changes, while polymers with rather small $\kappa_{b}$ go through significant conformational changes, but their bending energies are similar. This is exemplified in fig. 10, where $U_{b} /\left(k_{B} T\right)$ is shown for various bending rigidities and $P e=360$.

In figs. 9 and 10, the radial energy profiles are qualitatively similar for all $\kappa_{b}$ (with $L_{p} / L_{r}>1$ ) for a given Pe. For $0.5 \lesssim L_{p} / L_{r} \lesssim 5$, the polymer adopts Ushaped conformations at the center and hence $U_{b}$ is large. Away from the center, $U_{b}$ decreases as U-shapes disappear. With increasing radial center-of-mass position, the local shear rate increases and a polymer with small persistence length assumes transient bent conformations. The average over individual configurations provides a 

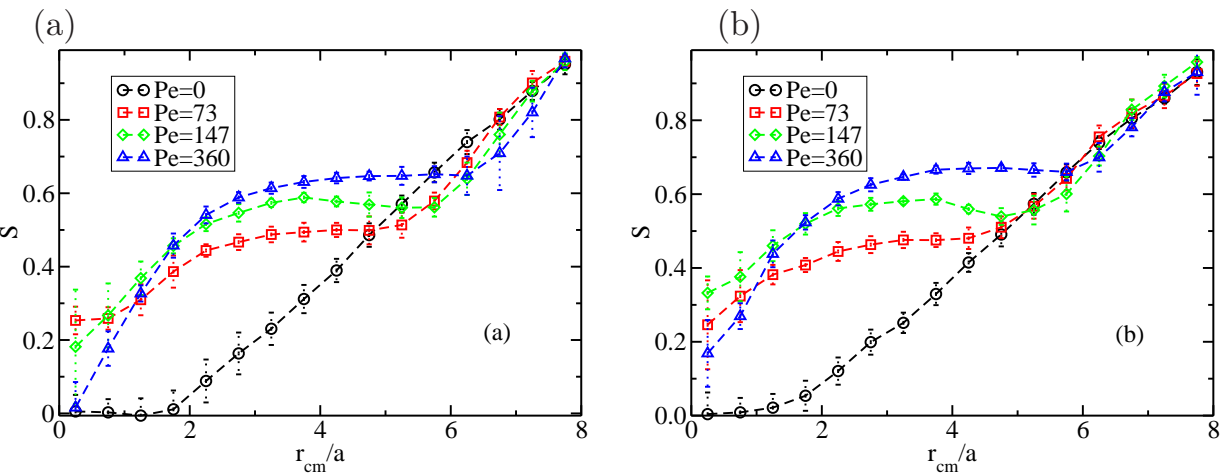

(c)

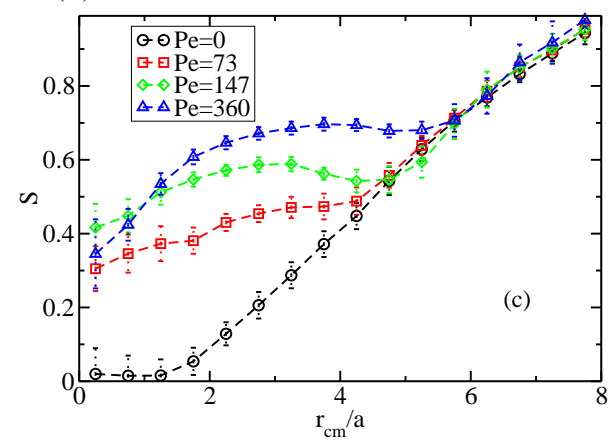

(d)

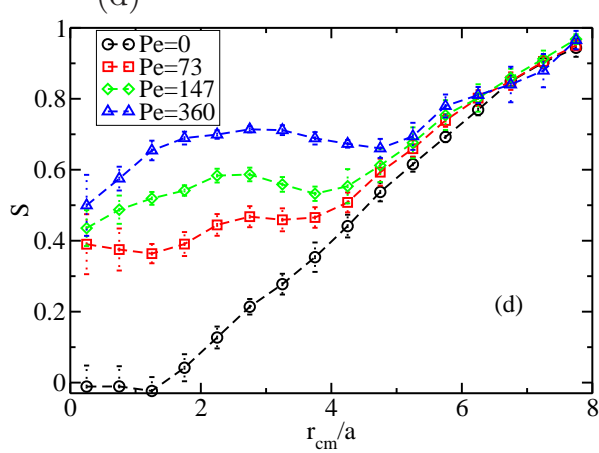

Figure 7. Orientation order parameter for the Peclet numbers $P e=0$ (०), 73 $(\square), 147(\diamond)$, and $360(\triangle)$ of polymers with $L_{p} / L_{r} \approx 3.8(\mathrm{a}), 7.7$ (b), 15.4 (c), and $30.8(\mathrm{~d})$.

(a)

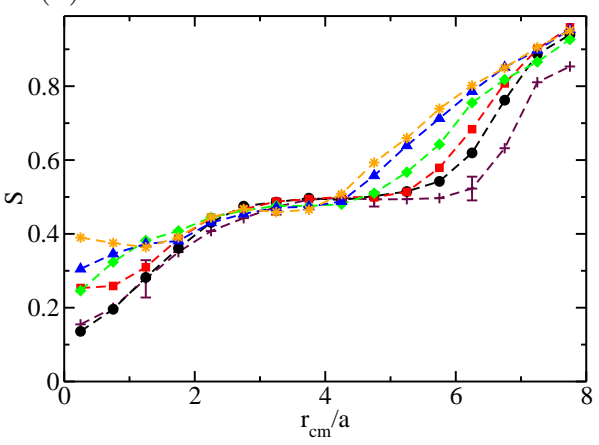

(b)

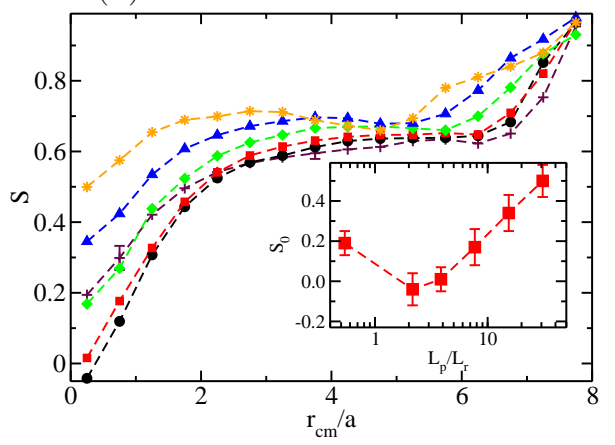

Figure 8. Orientational order parameters $S$ for Peclet numbers $P e=73$ (a) and 360 (b) and persistence lengths $L_{p} / L_{r} \approx 0.5(+), 2.1(\bullet), 3.8(\square), 7.7(\diamond), 15.4$ $(\triangle)$, and $30.8(\star)$. Inset: $S$ close to the channel center for $P e=360$. The line is a guide to the eye. 
(a)

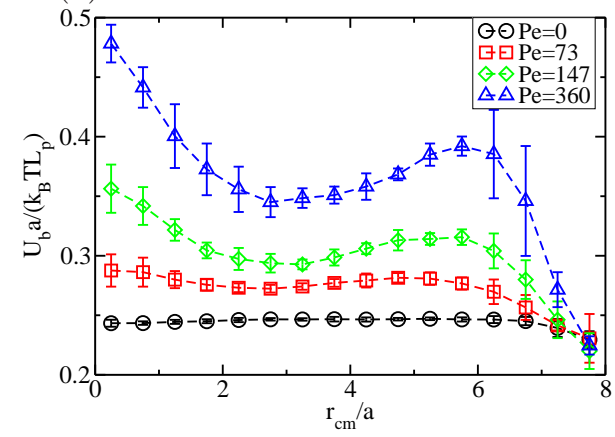

(b)

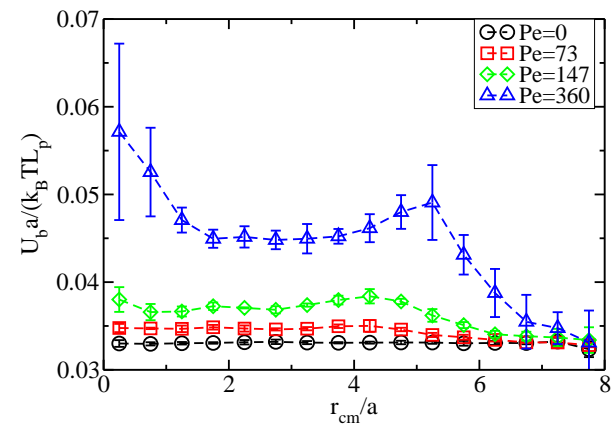

Figure 9. Bending energies $U_{b}$ for Peclet numbers $P e=0$ (०), $73(\square), 147(\diamond)$, and $360(\triangle)$ of polymers with persistence lengths $L_{p} / L_{r} \approx 3.8$ (a) and $30.8(\mathrm{~b})$.

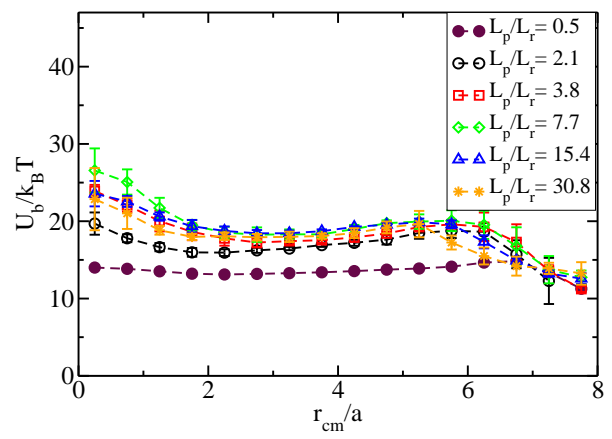

Figure 10. Bending energies for Peclet number $P e=360$ and persistence lengths $L_{p} / L_{r} \approx 0.5(\bullet), 2.1(\square), 3.8(\diamond), 7.7(\triangle), 15.4(\star)$, and $30.8(+)$.

high value for $U_{b}$ for such $r_{c m}$. The extent of bending decreases as $\kappa_{b}$ is increased, since for stiffer polymers $(1-\cos \vartheta) \sim U_{b} / \kappa_{b}$, where $\vartheta$ is the angle between successive bond vectors. More aligned bonds and hence smaller angle $\vartheta$ imply that $U_{b} / \kappa_{b}$ decreases with increasing $\kappa_{b}$, in agreement with the results of fig. 9 .

\section{Structural Properties without Hydrodynamic Interactions}

As is generally accepted by now, hydrodynamic interactions determine the behavior of polymers in microcapillary flows. In order to separate effects due to intramolecular and surface hydrodynamic interactions from those caused by the flow and steric interactions, we performed Brownian MPC simulations 73. Brownian dynamics simulation have also been performed in Ref. [57, with a polymer composed of rodlike segments. However, intramolecular hydrodynamic interactions are taken into account by employing anisotropic drag coefficients parallel and perpendicular to the rodlike segments. In contrast, our polymer model is composed of point-like monomers with isotropic friction. Hence, we consider a free draining chain, whereas in Ref. [57] intramolecular hydrodynamic interactions are taken into account implicitly. 
(a)

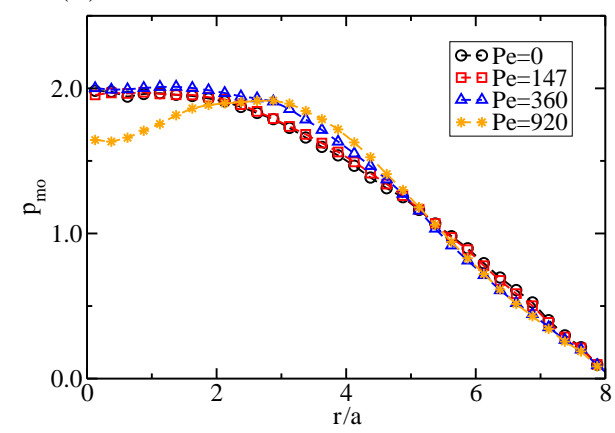

(b)

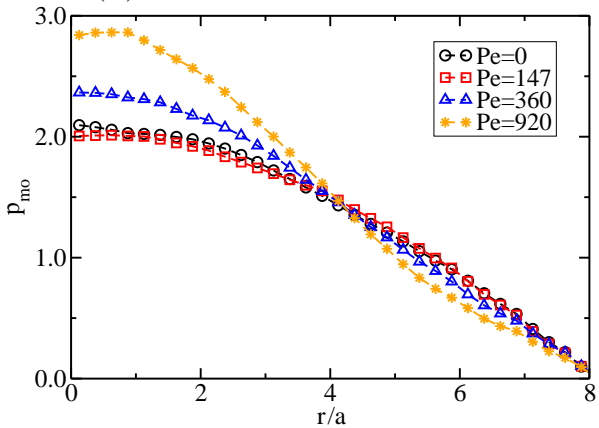

Figure 11. Radial monomer distributions for systems without hydrodynamic interactions. The persistence lengths are $L_{p} / L_{r}=3.8$ (a) and $30.8(\mathrm{~b})$, and the Peclet numbers $P e=0(\circ), 147(\square), 360(\triangle)$, and $920(\star)$.

(a)

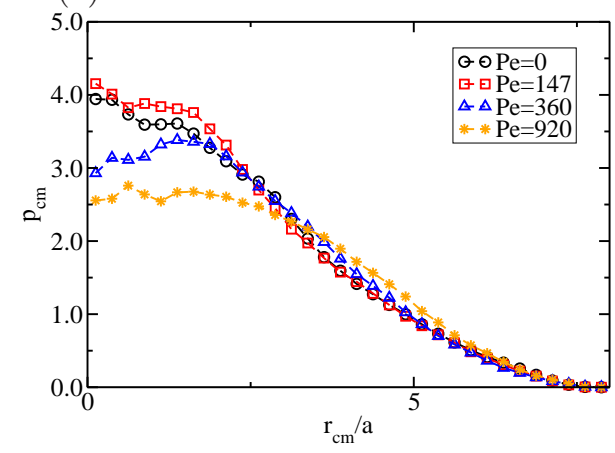

(b)

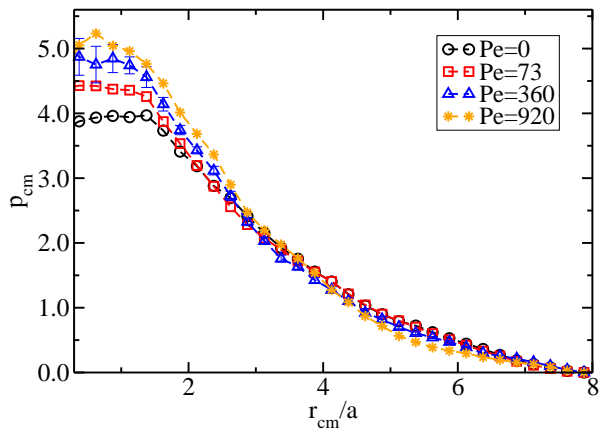

Figure 12. Radial center-of mass distributions for systems without hydrodynamic interactions. The persistence lengths are $L_{p} / L_{r}=3.8$ (a) and 30.8 (b) and the Peclet numbers $P e=0(\circ), 147(\square), 360(\triangle)$, and $920(\star)$.

\subsection{Radial Concentration Distributions}

The radial monomer probability distributions for the persistence lengths $L_{p} / L_{r} \approx 2.1$ and 30.8 are presented in fig. 11] for various flow rates. For $P e<200$, the distribution functions are rather similar. Adjacent to the wall, the distributions for small stiffnesses are even similar for all flow rates. At large flow rates and persistence lengths $L_{p} / L_{r}<3.5$, we find a monomer density increase at $r_{c m} / a \approx 4$, which we attribute to the induced alignment of the polymers by the flow. However, for larger stiffnesses, we find an inward migration adjacent to the wall due to increased steric polymer-surface interactions (cf. fig. 11b). Here, the polymer tumbling motion combined with the smaller number of conformational degrees of freedom at large stiffnesses leads to a strong repulsion from the wall. At the same time, the density in the channel center increases with a maximum at $r \approx 0$.

The radial center-of-mass distribution exhibits an outward migration even without hydrodynamic interactions for $L_{p} / L_{r}<3.5$ due to polymer alignment (cf. fig. 12). In contrast, $P_{c m}$ becomes narrower with increasing flow rate for $L_{p} / L_{r} \approx 30.8$, similar 
to the monomer distribution. Hence, a qualitative different behavior is obtained depending on the ability of the polymer to adjust to the conformational restrictions by the walls.

The Brownian simulations of ref. [57] display in strong outward migration of the polymers, which leads to a density minimum at the channel center and a large density increase near the walls. Two effects contribute to this behavior. On the one hand alignment of the polymers and on the other hand anisotropic diffusion by the aligned segments. We do not observe such an increase in density adjacent to the wall, because no hydrodynamic effects are included in our simulations.

\subsection{Alignment}

The orientational order parameters display qualitatively similar dependencies on flow rate as those for systems with hydrodynamic interactions (cf. figs. 7 and 8). However, alignment is far less pronounced and much larger Peclet numbers are required to achieve significant flow alignment. For $P e=360$, we find $S \approx 0.2$ at $L_{p} / L_{r} \approx 3.8$, compared to $S=0.6$ in the presence of hydrodynamic interactions. At the same time, we also observe the appearance of U-shaped structures in the channel center for larger Peclet numbers. The comparison shows that certain qualitative features of polymer alignment are determined by the flow profile, rather than hydrodynamic interactions. However, hydrodynamic interactions clearly influence the polymer orientation in a quantitative manner and lead to a more pronounced alignment.

\section{Cross-Streamline Dynamics}

The most striking effect of flow on a semiflexible polymer is the strong dependence of its orientation on $P e$ and the radial distance. This aspect provides the key to understand the appearance of the off-center maximum in the center-of-mass distribution function.

As is well known, a rod in solution exhibits a larger diffusion coefficient parallel to its axis than perpendicular to it. In the absence of flow, a semiflexible polymer behaves very similar to a stiff rod, we therefore expect that the observed polymer orientational differences across the channel will lead to differences in the lateral diffusion behavior.

In order to characterize this diffusive behavior, we calculate the radial mean displacement $\Delta r\left(r_{c m}\right)=\left\langle\left[\boldsymbol{r}_{c m}\left(t_{0}+\Delta t\right)-\boldsymbol{r}_{c m}\left(t_{0}\right)\right] \boldsymbol{e}_{c m}\left(t_{0}\right)\right\rangle$, where $\boldsymbol{e}_{c m}=$ $\boldsymbol{r}_{c m} /\left|\boldsymbol{r}_{c m}\right|$, and the center-of-mass mean square displacement $\Delta r^{2}\left(r_{c m}\right)=$ $\left\langle\left(\boldsymbol{r}_{c m}\left(t_{0}+\Delta t\right) \boldsymbol{e}_{c m}\left(t_{0}\right)-\left\langle\boldsymbol{r}_{c m}\left(t_{0}\right) \boldsymbol{e}_{c m}\left(t_{0}\right)\right\rangle\right)^{2}\right\rangle$ within a certain time $\Delta t$ for a polymer with the center-of-mass position $\boldsymbol{r}_{c m}$ at $t_{0}$. We choose the time $\Delta t$ such that the center-of-mass displacement is smaller than $a$ and the mean square displacement is linear in time. For an homogeneous and isotropic system, $\Delta r$ and $\Delta r^{2}$ are independent of the initial position.

The mean values are the first and second moment of the conditional probability distribution function to find the polymer center-of-mass at position $r_{c m}\left(t_{0}+\Delta t\right)$ at the time $t_{0}+\Delta t$, when it has been at position $r_{c m}\left(t_{0}\right)$ at the time $t_{0}$. Initially, the distribution is very narrow, ideally a $\delta$ function, and broadens in time. In the limit $\Delta t \rightarrow \infty$ the conditional probability distribution turns into the equilibrium distribution function as displayed in figs. 4 and 5 .

Figures 13 and 14 display $\Delta r$ and $\Delta r^{2}$ for various Peclet numbers and stiffnesses. The mean displacements evidently depend on flow rate and stiffness. At $P e=0$, there is no drift in the channel center. Only for $r_{c m} / a \gtrsim 1.5$, we observe a drift, which 

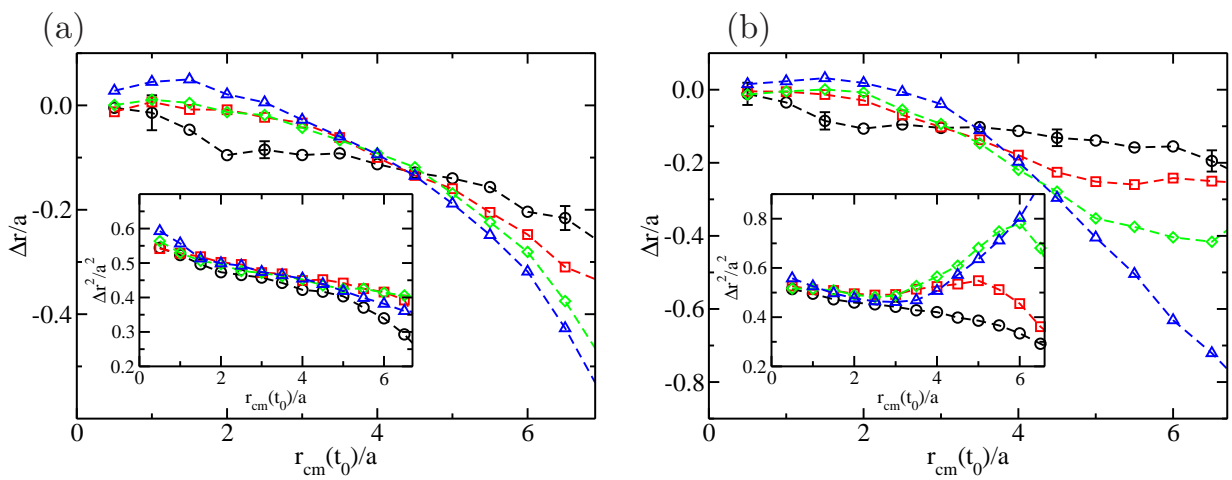

Figure 13. Radial center-of-mass displacements $\Delta r\left(r_{c m}\right)$ and mean square displacements $\Delta r^{2}\left(r_{c m}\right)$ (insets) for Peclet numbers $P e=0$ (o), $73(\square), 147$ $(\diamond)$, and $360(\triangle)$ of polymers with persistence lengths $L_{p} / L_{r} \approx 3.8$ (a) and 30.8 (b).

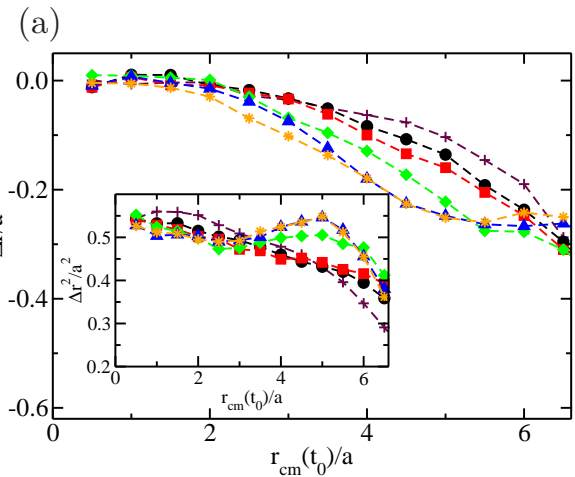

(b)

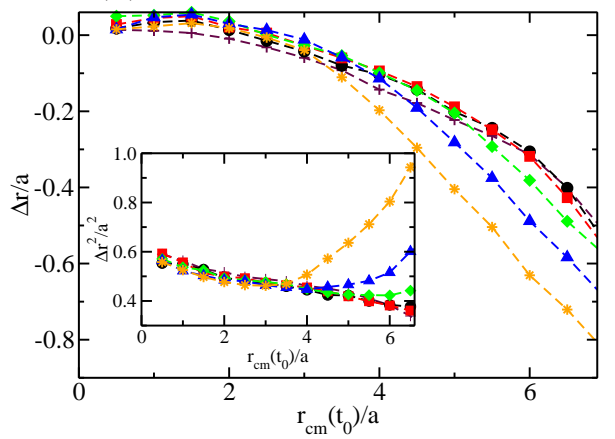

Figure 14. Radial center-of-mass displacements $\Delta r\left(r_{c m}\right)$ and mean square displacements $\Delta r^{2}\left(r_{c m}\right)$ (insets) for persistence lengths $L_{p} / L_{r} \approx 0.5(+), 2.1$ $(\bullet), 3.8(\square), 7.7(\diamond), 15.4(\triangle)$, and $30.8(\star)$, and $P e=73(\mathrm{a})$ and $P e=360(\mathrm{~b})$.

we attribute to steric polymer wall interactions, and which increase adjacent to the wall. For larger flow rates, the drift for $r_{c m} \lesssim 4$ is close to zero due to alignment of a polymer and lack of steric wall interactions (cf. fig. 14a). At large Peclet numbers, $\Delta r$ is positive for $r_{c m} / a<2.5$ (cf. fig. 14b) and all persistence lengths $L_{p} / L_{r}>2$, which indicates an outward migration. The wall interactions lead to a larger drift of polymers towards the channel center adjacent to the wall, which increases with increasing stiffness.

The mean square displacement decreases with increasing radial distance for $r_{c m}<4 a$ at all stiffnesses and flow rates. Since $\Delta r$ is rather small for $r_{c m}<4 a$, $\Delta r^{2}$ is determined by diffusion, i.e., the radial diffusion is larger in the channel center. This leads to a minimum in the center-of-mass distribution at large flow rates and stiffnesses. At larger radial distances and large stiffnesses, the mean square displacement is strongly affected by wall interactions. There is no simple explanation for the non-monotonic behavior of the mean square displacement. Various wall interactions contribute to the system behavior: the wall lift force, steric wall 


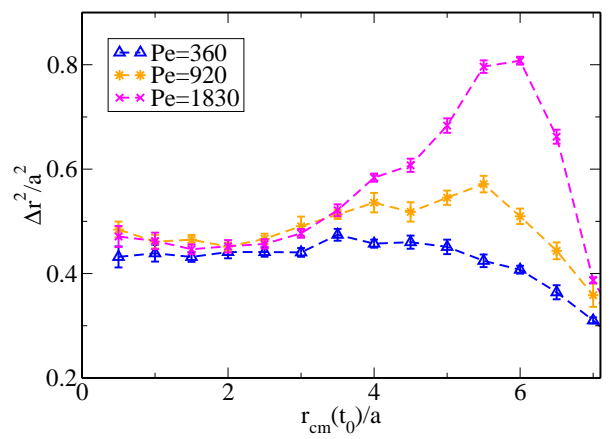

Figure 15. Radial center-of-mass mean square displacements without hydrodynamic interactions for the persistence lengths $L_{p} / L_{r} \approx 30.8$ and the Peclet numbers $P e=360(\triangle), 920(\star)$, and $1830(\times)$.

interactions, and an increase of the tumbling frequency with increasing flow rate [74,75].

Without hydrodynamic interactions, the drift at $P e=0$ is comparable to that of the system with hydrodynamic interactions. It changes with increasing Peclet number due to flow alignment, but only slightly. The mean square displacement is independent of the radial position for distances $r_{c m}<6 a$ for all Peclet numbers $P e<1000$ and stiffnesses as shown in fig.15. Only close to the wall, there is a decrease due to steric wall interactions. This supports our conclusion that intramolecular hydrodynamic interactions are responsible for outward migration in systems with hydrodynamic interactions. At much larger Peclet numbers $(P e \approx 2000)$, the mean square displacement is constant for $r<4 a$, increases then with the radial distance and decreases again beyond $r=6 a$, similar to the behavior shown in the inset of fig. 14 for $L_{p} / L_{r}=15.4$. This behavior is explained by the strong steric polymer wall interaction and fast tumbling motion at high flow rates. Note that the tumbling frequency increases with the shear rate [74,75]. Hence, systems with and without hydrodynamic interactions exhibit similar features, but larger Peclet numbers are required for systems without hydrodynamic interactions and the behavior of systems with hydrodynamic interactions is richer and additional features are displayed.

\section{Summary and Conclusions}

We have analyzed the flow behavior of semiflexible polymers confined in a cylindrical channel and systematically studied the influence of bending rigidity on structural properties and cross-streamline migration. The study includes polymers with moderate bending rigidity, comparable to actin filaments, to very stiff rodlike polymers.

Poiseuille flow induces a strong radial-dependent polymer alignment parallel to the channel associated with a radially outward migration of its center-of-masses at small Peclet numbers and, at large Peclet numbers, a wall induces inward migration.

Stiffness changes the polymer behavior qualitatively and quantitatively. Taking a system without hydrodynamic interactions as a reference, there is no outward migration and there are no wall hydrodynamic forces. Correspondingly, the centerof-mass density decreases in the channel center with increasing flow rate and up to 
a certain stiffness $\left(L_{p} / L_{r}<20\right)$ due to polymer alignment. For large stiffnesses, however, steric polymer-wall interactions cause inward migration of the molecule and an increase in density at the channel center with increasing flow rate. We attribute this effect to the increase of the tumbling frequency at large flow rates and hence more polymer-wall contacts. Hence, even for such a system, a qualitative different behavior is found for large and small stiffnesses.

This picture is enriched by hydrodynamic interactions, which enhance outward migration. At the same time wall-lift forces lead to inward migration and an offcenter density maximum is formed at large flow rates. Such a maximum has also been found in experiments using actin filaments [47. The center-of-mass distribution strongly depends on stiffness and the presence of hydrodynamic interactions, as is reflected in its width. Flexible polymers exhibit a more complex migration behavior than stiffer polymers due to conformational changes, i.e, stretching and alignment. For large stiffnesses, wall steric interactions lead to a strong inward migration as for polymers without hydrodynamic interactions. The semiflexible polymers exhibit a non-universal behavior when changing stiffness and flow rate. At low flow rates, stiffer polymers display a stronger inward migration, whereas at high flow rates a reversed trend is observed initially and ultimately a strong inward migration at large stiffnesses. It is the interplay between confinement, hydrodynamic interactions, and stiffness which leads to the emergence of a complex structural and dynamical behavior of the polymer. This is reflected in the stiffness dependence of the mean radial and mean square radial displacements.

Although the understanding of the flow behavior of soft mesoscale particles in nano- and microchannels has made considerable progress in recent years, this field can be expected to attract far more attention in the future. For example, flow in structured channels has hardly been addressed so far. Also, interactions between polymers at finite concentration, and between polymers and other soft particles, needs to be investigated.

\subsection{Acknowledgments}

The financial support and the stimulating environment of the DFG priority program "Nano- and Microfluidics" is gratefully acknowledged. In particular, we thank R. Finken and U. Seifert (Stuttgart), L. Schmid and T. Franke (Augsburg), and D. Steinhauser and T. Pfohl (Göttingen \& Basel) for many helpful and inspiring discussions.

\section{References}

[1] F. T. Wall, W. A. Seitz, J. C. Chin, and P. G. de Gennes. Statistics of self-avoiding walks confined to strips and capillaries. Proc. Natl. Acad. Sci. USA, 75:2069, 1978.

[2] F. Brochard and P. G. de Gennes. Dynamics of confined polymer chains. J. Chem. Phys., 67:52, 1977.

[3] J. H. van Vliet and G. ten Brinke. Orientation and shape of flexible polymers in a slit. J. Chem. Phys., 93:1436-1441, 1990.

[4] R. M. Jendrejack, E. T. Dimalanta, D. C. Schwartz, M. D. Graham, and J. J. De Pablo. Dna dynamics in a microchannel. Phys. Rev. Lett., 91:038102, 2003.

[5] A. Balducci, C.-C. Hsieh, and P. S. Doyle. Relaxation of stretched dna in slitlike confinement. Phys. Rev. Lett., 99:238102, 2007.

[6] R. M. Jendrejack, D. C. Schwartz, M. D. Graham, and J. J. de Pablo. Effect of confinement on dna dynamics in microfluidic devices. J. Chem. Phys., 119:1165, 2003. 
[7] J. O. Tegenfeldt, C. Prinz, H. Cao, W. W. Reisner, R. Riehn, Y. M. Wang, E. C. Cox, J. C. Sturm, P. Silberzan, and R. H. Austin. The dynamics of genomic-length dna molecules in 100-nm channels. Proc. Natl. Acad. Sci. USA, 101:10979-10983, 2004.

[8] U. S. Agarwal, A. Dutta, and R. A. Mashelkar. Migration of macromolecules under flow: the physical origin and engineering implications. Chem. Eng. Sci., 49:1693, 1994.

[9] R. M. Jendrejack, D. C. Schwartz, J. J. de Pablo, and M. D. Graham. Shear-induced migration in flowing polymer solutions: Simulation of long-chain dna in microchannels. J. Chem. Phys., 120:2513, 2004 .

[10] H. Noguchi and G. Gompper. Shape transitions of fluid vesicles and red blood cells in capillary flows. Proc. Natl. Acad. Sci. USA, 102:14159-14164, 2005.

[11] O. B. Usta, J. E. Butler, and A. J. C. Ladd. Flow-induced migration of polymers in dilute solution. Phys. Fluids, 18:031703, 2006.

[12] R. Khare, M. D. Graham, and J. J. de Pablo. Cross-stream migration of flexible molecules in a nanochannel. Phys. Rev. Lett., 96:224505, 2006.

[13] D. Stein, F. H. J. van der Heyden, W. J. A. Koopmans, and C. Dekker. Pressure-driven transport of confined dna polymers in fluid channels. Proc. Natl. Acad. Sci. USA, 103:15853-15858, 2006.

[14] O. B. Usta, J. E. Butler, and A. J. C. Ladd. Transverse migration of a confined polymer driven by an external force. Phys. Rev. Lett., 98:098301, 2007.

[15] L. Cannavacciuolo, R. G. Winkler, and G. Gompper. Mesoscale simulations of polymer dynamics in microchannel flows. EPL, 83:34007, 2008.

[16] A. P. Markesteijn, O. B. Usta, I. Ali, A. C. Balazsac, and J. M. Yeomans. Flow injection of polymers into nanopores. Soft Matter, 5:4575, 2009.

[17] J. Liam McWhirter, Hiroshi Noguchi, and Gerhard Gompper. Flow-induced clustering and alignment of red blood cells in microchannels. Proc. Natl. Acad. Sci. USA, 106:6039-6043, 2009.

[18] Hiroshi Noguchi, Gerhard Gompper, Lothar Schmid, Achim Wixforth, and Thomas Franke. Dynamics of fluid vesicles in flow through structured microchannels. EPL, 89:28002, 2010.

[19] Todd M. Squires and Stephen R. Quake. Microfluidics: Fluid physics at the nanoliter scale. Rev. Mod. Phys., 77:977-1026, 2005.

[20] G. Whitesides. The origins and the future of microfluidics. Nature, 442:403, 2006.

[21] Harold Craighead. Future lab-on-a-chip technologies for interrogating individual molecules. Nature, 442:387-393, 2006.

[22] R. Skalak. Deformation of red blood cells in capillaries. Science, 164:717-719, 1969.

[23] T. W. Secomb, R. Skalak, N. Özkaya, and J. F. Gross. Flow of axisymmetric red blood cells in narrow capillaries. J. Fluid Mech., 163:405-423, 1986.

[24] A. R. Pries, T. W. Secomb, and P. Gaehtgens. Biophysical aspects of blood flow in microvasculature. Cardiovasc. Res., 32:654-667, 1996.

[25] C. Pozrikidis. Axisymmetric motion of a file of red blood cells through capillaries. Phys. Fluids, 17:031503, 2005.

[26] R. G. Blazej, P. Kumaresan, and R. A. Mathies. Microfabricated bioprocessor for integrated nanoliter-scale sanger dna sequencing. Proc. Natl. Acad. Sci. USA, 103:7240-7245, 2006.

[27] A. S. Utada, L.-Y. Chu, A. Fernandez-Nieves, D. R. Link, C. Holtze, and D. A. Weitz. Dripping, jetting, drops, and wetting: The magic of microfluidics. MRS Bull., 32:702-708, 2007.

[28] W. Dzwinel, K. Boryczko, and D. A. Yuen. A discrete-particle model of blood dynamics in capillary vessels. J. Colloid Int. Sci., 258:163-173, 2003.

[29] H. Noguchi and G. Gompper. Fluid vesicles with viscous membranes in shear flow. Phys. Rev. Lett., 93:258102, 2004.

[30] Michael M. Dupin, Ian Halliday, Chris M. Care, Lyuba Alboul, and Lance L. Munn. Modeling the flow of dense suspensions of deformable particles in three dimensions. Phys. Rev. E, 75:066707, 2007.

[31] Reimar Finken, Antonio Lamura, Udo Seifert, and Gerhard Gompper. Two-dimensional fluctuating vesicles in linear shear flow. Eur. Phys. J. E, 25:309-321, 2008.

[32] Manouk Abkarian, Magalie Faivre, Renita Horton, Kristian Smistrup, Catherine A. BestPopescu, and Howard A. Stone. Cellular-scale hydrodynamics. Biomed. Mater., 3:034011, 2008.

[33] Giovanna Tomaiuolo, Marino Simeone, Vincenzo Martinelli, Bruno Rotoli, and Stefano Guido. Red blood cell deformation in microconfined flow. Soft Matter, 5:3736-3740, 2009.

[34] V. Vitkova, M. Mader, and T. Podgorski. Deformation of vesicles flowing through capillaries. Europhys. Lett., 68:398-404, 2004.

[35] Hou-Pu Chou, Charles Spence, Axel Scherer, and Stephen Quake. A microfabricated device for 
sizing and sorting dna molecules. Proc. Natl. Acad. Sci. USA, 96:11-13, 1999.

[36] Stephen L. Levy and Harold G. Craighead. Dna manipulation, sorting, and mapping in nanofluidic systems. Chem. Soc. Rev., 39:1133-1152, 2010.

[37] Fredrik Persson and Jonas O. Tegenfeldt. Dna in nanochannels - directly visualizing genomic information. Chem. Soc. Rev., 39:985-999, 2010.

[38] T. T. Perkins, D. E. Smith, R. G. Larson, and S. Chu. Stretching of a single tethered polymer in a uniform flow. Science, 268:83-87, 1995.

[39] D. E. Smith, H. P. Babcock, and S. Chu. Single-polymer dynamics in steady shear flow. Science, $283: 1724,1999$

[40] W. Reisner, K. J. Morton, R. Riehn, Y. M. Wang, Z. Yu, M. Rosen, J. C. Sturm, S. Y. Chou, E. Frey, and R. H. Austin. Statics and dynamics of single dna molecules confined in nanochannels. Phys. Rev. Lett., 94:196101, 2005.

[41] C. M. Schroeder, R. E. Teixeira, E. S. G. Shaqfeh, and S. Chu. Characteristic periodic motion of polymers in shear flow. Phys. Rev. Lett., 95:018301, 2005.

[42] S. Gerashchenko and V. Steinberg. Statistics of tumbling of a single polymer molecule in shear flow. Phys. Rev. Lett., 96:038304, 2006.

[43] E. P. Petrov, T. Ohrt, R. G. Winkler, and P. Schwille. Diffusion and segmental dynamics of double-stranded dna. Phys. Rev. Lett., 97:258101, 2006.

[44] S. Köster, D. Steinhauser, and T. Pfohl. Brownian motion of actin filaments in conning microchannels. J. Phys. Condens. Matter, 17:S4091-S4104, 2005.

[45] S. Köster, J. Kierfeld, and T. Pfohl. Characterization of single semiflexible filaments under geometric constraints. Eur. Phys. J. E, 25:439-449, 2008.

[46] S. Köster and T. Pfohl. An in vitro model system for cytoskeletal confinement. Cell Motil. Cytoskeleton, 66:771-776, 2009.

[47] D. R. Steinhauser. Actin filaments and bundles in flow. PhD Thesis, University Göttingen, Germany, 2008.

[48] T. Odijk. On the statistics and dynamics of confined or entangled stiff polymers. Macromolecules, 16:1340-1344, 1983.

[49] Yingzi Yang, Theodore W. Burkhardt, and Gerhard Gompper. Free energy and extension of a semiflexible polymer in cylindrical confining geometries. Phys. Rev. E, 76:011804, 2007.

[50] P. Levi and K. Mecke. Radial distribution function for semiexible polymers conned in microchannels. Europhys. Lett., 78:38001, 2007.

[51] T. Odijk. Scaling theory of dna confined in nanochannels and nanoslits. Phys. Rev. E, 77:060901(R), 2008.

[52] G. Gompper and D. M. Kroll. Driven transport of fluid vesicles through narrow pores. Phys. Rev. E, 52:4198-4208, 1995.

[53] A. P. Sakaue, E. Raphael, P. G. de Gennes, and F. Brochard-Wyart. Flow-injection of branched polymers inside nanopores. Europhys. Lett., 72:83-88, 2005.

[54] G. T. Linke, R. Lipowsky, and T. Gruhn. Osmotically induced passage of vesicles through narrow pores. Europhys. Lett., 74:916-922, 2006.

[55] C. Sendner and R. R. Netz. Shear-induced repulsion of a semiflexible polymer from a wall. $E P L, 81: 54006,2008$

[56] R. Schiek and E. S. G. Shaqfeh. Cross-streamline migration of slender brownian fibres in plane poiseuille flow. J. Fluid. Mech., 332:23, 1997.

[57] D. Saintillan, E. S. G. Shaqfeh, and E. Darve. Effect of flexiblity on the shear-induced migration of short-chain polymers in parabolic channel flow. J. Fluid. Mech., 557:297, 2006.

[58] K. Mussawisade, M. Ripoll, R. G. Winkler, and G. Gompper. Dynamics of polymers in a particle based mesoscopic solvent. J. Chem. Phys., 123:144905, 2005.

[59] R. G. Winkler, K. Mussawisade, M. Ripoll, and G. Gompper. Rodlike colloids and polymers in shear flow: A multi-particle-collision dynamics study. J. Phys. Condens. Matter, 16:S3941S3954, 2004.

[60] Movies are available as Supporting Material at the IOP-Website [???????]

[61] A. Malevanets and R. Kapral. Mesoscopic model for solvent dynamics. J. Chem. Phys., 110:8605-8613, 1999

[62] A. Malevanets and R. Kapral. Solute molecular dynamics in a mesoscopic solvent. J. Chem. Phys., 112:7260-7269, 2000.

[63] R. Kapral. Multiparticle collision dynamics: simulations of complex systems on mesoscale. Adv. Chem. Phys., 140:89, 2008.

[64] G. Gompper, T. Ihle, D. M. Kroll, and R. G. Winkler. Multi-particle collision dynamics: A particle-based mesoscale simulation approach to the hydrodynamics of complex fluids. Adv. Polym. Sci., 221:1, 2009. 
[65] M. Ripoll, K. Mussawisade, R. G. Winkler, and G. Gompper. Low-Reynolds-number hydrodynamics of complex fluids by multi-particle-collision dynamics. Europhys. Lett., 68:106, 2004.

[66] M. Ripoll, K. Mussawisade, R. G. Winkler, and G. Gompper. Dynamic regimes of fluids simulated by multi-particle-collision dynamics. Phys. Rev. E, 72:016701, 2005.

[67] A. Lamura, G. Gompper, T. Ihle, and D. M. Kroll. Multiparticle collision dynamics: Flow around a circular and a square cylinder. Europhys. Lett., 56:319-325, 2001.

[68] A. Malevanets and J. M. Yeomans. Dynamics of short polymer chains in solution. Europhys. Lett., 52:231-237, 2000.

[69] M. A. Webster and J. M. Yeomans. Modelling a tethered polymer in poiseuille flow. J. Chem. Phys., 122:164903, 2005.

[70] C.-C. Huang, A. Chatterji, G. Sutmann, G. Gompper, and R. G. Winkler. Cell-level canonical sampling by velocity scaling for multiparticle collision dynamics simulations. J. Comput. Phys., 229:168, 2010.

[71] M. Hecht, J. Harting, T. Ihle, and H. J. Herrmann. Simulation of claylike colloids. Phys. Rev. E, 72:011408, 2005

[72] N. Kikuchi, C. M. Pooley, J. F. Ryder, and J. M. Yeomans. Transport coefficients of a mesoscopic fluid dynamics model. J. Chem. Phys., 119:6388-6395, 2003.

[73] M. Ripoll, R. G. Winkler, and G. Gompper. Hydrodynamic screening of star polymers in shear flow. Eur. Phys. J. E, 23:349, 2007.

[74] R. G. Winkler. Semiflexible polymers in shear flow. Phys. Rev. Lett., 97:128301, 2006.

[75] R. Chelakkot, R. G. Winkler, and G. Gompper. Migration of semiflexible polymers in microcapillary flow. EPL, 91:14001, 2010. 